\title{
Estudio Comparativo sobre Percepción y uso de las TIC entre Profesores de Universidades Públicas y Privadas
}

\author{
Omar A. Tapasco ${ }^{(1,2)}$ y Jaime A. Giraldo ${ }^{(2)}$ \\ (1) Universidad de Caldas, Cl 65 No. 26.10, Manizales, Colombia. (e-mail: omar.tapasco@ucaldas.edu.co) \\ (2) Universidad Nacional de Colombia sede Manizales, Cr. 27 No. 64-60, Manizales, Colombia \\ (e-mail: jaiagiraldo@unal.edu.co)
}

Recibido Jul. 14, 2016; Aceptado Sep. 23, 2016; Versión final Nov. 25, 2016, Publicado Abr. 2017

\begin{abstract}
Resumen
La presente investigación se propuso recoger información sobre el uso de las Tecnologías de la Información y la Comunicación (TIC) en la actividad docente universitaria, la percepción del profesorado sobre las TIC y la valoración de la infraestructura tecnológica disponible, desde una perspectiva comparativa entre universidades públicas y privadas. Para tal propósito se realizó una encuesta entre 147 profesores de instituciones del Eje Cafetero, Colombia. Se recurrió al análisis factorial, el análisis de correspondencias múltiples y pruebas de independencia. Se encontraron marcadas diferencias entre profesores de universidades públicas y privadas. Algunas diferencias con respecto al género, pero no se hallaron diferencias significativas con respecto a otras variables descriptivas del docente como la edad, la escolaridad o los años de experiencia docente. Pero aunque los indicadores favorecen a los docentes de instituciones privadas, también se evidencia un mayor nivel de estrés asociado al uso de las tecnologías entre ellos.
\end{abstract}

Palabras clave: uso de las TIC; percepción sobre las TIC; universidades públicas y privadas; análisis factorial; análisis de correspondencias múltiples

\section{Comparative Study about Perception and use of ICT among Teachers of Public and Private Universities}

\begin{abstract}
The purpose of this research was to collect information about the use of Information and Communication Technologies (ICT) in university teaching, the perception of the teachers about the ICT and the assessment of the technological infrastructure available, from a comparative perspective between public and private universities. For this, a survey was carried out among 147 teachers from institutions of the Eje Cafetero, Colombia. Factor analysis, multiple correspondence analysis and independence tests were used. Marked differences were found between teachers of public and private universities, some differences with respect to gender, but no significant differences were found in relation to other descriptive variables of the teacher such as age, education level or years of teaching experience. But although the results are favorable to the teachers of private institutions, there is also evidence of a higher level of stress associated with the use of technologies among them.
\end{abstract}

Keywords: use of ICT, perception about ICT; public and private universities; factorial analysis; multiple correspondence analysis 


\section{INTRODUCCIÓN}

Según el Reporte Global de Tecnologías de la Información, la calificación obtenida para Colombia en el 2015 fue de 4.1 en una escala de uno a siete, resultado que ubica al país en el puesto 64 de 143 economías, superando al $55.2 \%$ de los países evaluados, que desagregado por subíndices registra valores muy heterogéneos, en términos de países que supera en cada ítem, a saber: "Entorno de las $\mathrm{TIC}$ " $32.2 \%$, "Disponibilidad de las TIC" 58.7\%, "Uso de las TIC" 58.7\% e "Impacto de la TIC" 63.6\%. En el contexto latinoamericano se ubica quinto después de Chile, Uruguay, Costa Rica y Panamá, pero por encima de países referentes como Brasil, México y Argentina (DNP Colombia, 2015). Y según datos del Ministerio de Educación Nacional de Colombia (2016) más del $80 \%$ de la matrícula escolar en educación básica y media del país tiene acceso a internet, con una proporción de cinco estudiantes por computador en la actualidad, pero evidenciándose grandes inequidades entre regiones y registrando indicadores ampliamente favorables a los centros urbanos.

Se evidencia además un gran dinamismo entre los países de la región hacia la adopción de las TIC, pero en el contexto de instituciones de educación superior la integración de las mismas se encuentra en una fase inicial, muy lejos aún del total aprovechamiento de las posibilidades que ellas ofrecen (Baelo y Cantón, 2009). Aunque es importante reseñar que la incorporación de innovaciones tecnológicas por sí mismas no conduce a un mejoramiento de las prácticas educativas, dado que con frecuencia se observa que las tecnologías han sido introducidas a la enseñanza universitaria sin considerar las implicaciones que ellas tienen sobre el aprendizaje del estudiante (Kirkwood y Price, 2006). Conduciendo con frecuencia a procesos sin sentido, como lo es el incorporar tecnologías para realizar las mismas labores que se hacen en entornos no tecnológicos (Cabero, 2005) o con acciones tales como el uso de las aulas virtuales sólo para transmisión de información, el uso de la tutoría como momento de control y no de crecimiento, la evaluación limitada a procesos sumativos, entre otros (Correa y Paredes, 2009).

Entre las dificultades para usar e integrar las TIC en la enseñanza, se señalan el hecho de que los profesores de hoy no hayan aprendido con las TIC cuando ellos eran estudiantes; el que los cursos tengan un enfoque hacia técnicas de enseñanza grupales en modo presencial y no hacia una enseñanza individual en línea, e igualmente le atribuyen incidencia al hecho de que tanto el hardware como el software educativo disponibles esté en constante cambio (Nicholas y Guzman 2009), lo que obliga al profesorado a estar igualmente inmerso en una continua formación (Morales et al., 2015). Unido a una debilidad detectada por la ausencia de cursos de capacitación que propicien el tránsito de una incorporación de las TIC en el modo presencial hacia otras modalidades virtuales o semi-presenciales (Lagunes et al., 2015).

Tomando en consideración a los alumnos, se debe tener presente que los jóvenes de hoy, nacidos y formados en la era digital (nativos digitales), requieren de un apoyo didáctico metodológico que les permitan hacer uso efectivo, eficiente y adecuado de la tecnología para su capacitación formal, dado que estos nativos digitales, entran a las instituciones educativas, a cualquier nivel de educación, con un dominio y competencia tecnológica muy significativa (Rosario y Vásquez 2012). Configurándose una situación paradójica, como el hecho de que las nuevas generaciones posean conocimientos no dominados por las antiguas (Cabero, 2005). Y consecuencia de ello puede ser que los métodos de enseñanza tradicional lleguen a ser cuestionados como un cambio en las expectativas. Por ejemplo, los estudiantes que están ahora familiarizados con formatos de comunicación a través de herramientas de aprendizaje social, por ejemplo, WhatsApp, Facebook o Twitter, esperan que estas sean replicadas en el salón de clases. Lo que ocasiona que el personal académico esté encarando nuevos ambientes de aprendizaje, generándose más complejidad y presión cuando ellos enseñan a estudiantes con una marcada diversidad (Rienties et al., 2013).

Desde la perspectiva del docente se tiene que a pesar de que la mayor parte de los usos que se hacen de las TIC se encuentran relacionados a una utilización básica, tales como acceso y presentación de la información, desarrollo de la comunicación, seguimiento de actividades, entre otras, siguen persistiendo importantes vacíos en cuanto a su utilización para actividades con una mayor exigencia de integración, tales como simulaciones, trabajo colaborativo, evaluación, y actividades de acompañamiento (Baelo 2011). Igualmente, Cabero et al. (2011) comentan que a pesar de que una alta proporción de docentes manifiesta conocer diversas aplicaciones ofimáticas de utilidad en el trabajo de clase, muy pocos se aventuran en el diseño de actividades en línea que complementen o apoyen los procesos de enseñanza y aprendizaje, por lo que se infiere la necesidad de una formación específica en estas áreas telemáticas. En palabras de Cantón y Baelo (2011), nos encontramos ante la paradoja de un profesorado que se cree capacitado para usar las TIC pero con dificultades para integrarlas, es decir, conoce su manejo, pero no encuentra la forma de hacerlas parte de sus actividades profesionales.

También es importante señalar la relevancia de los procesos de alfabetización digital para el uso de la información en línea conducentes a la construcción y aplicación del conocimiento (Muñoz et al., 2012), con un docente que propicie la interacción de estas herramientas con el conocimiento, permitiendo el desarrollo 
de estructuras mentales en sus estudiantes y siendo consciente de los beneficios y las dificultades que estas pueden tener para el proceso educativo (Riascos et al., 2012). A través de mediaciones pedagógicas diferentes, que implican moverse de una enseñanza mecánica y memorística hacia otras que fomenten la participación activa del estudiante, propicien el trabajo colaborativo y faciliten el aprendizaje autónomo (Ausín et al., 2016).

Pero para el éxito de tales procesos es necesario trascender más allá de los aspectos de disponibilidad de recursos y capacitación pedagógica, surgiendo con particular importancia los sentimientos, creencias y percepciones de los profesores, tanto en nuevos como en experimentados, acerca de su habilidad para desarrollar un rango de tareas computacionales (Jones, 2012). Y es que simultáneo al rápido crecimiento de los medios tecnológicos han aflorado también actitudes contrapuestas, encontrándose a los entusiastas de la tecnología, que ven en ella un nuevo paradigma de relaciones y de acceso a la información y el conocimiento, así como a los detractores del uso masivo de la tecnología, que ya sea por convicción o como excusa, se resisten al uso intensivo de la tecnología en sus vidas personal y profesional (Duart y Reparaz, 2016). Aunque es natural la resistencia de los usuarios ante los cambios; es relevante conocer las características particulares de los usuarios de las TIC, para poder diseñar estrategias que correspondan a la realidad particular de la sociedad a la que pertenecen (Yong et al., 2010).

Las diversas actitudes y percepciones de los docentes frente a las TIC dependen en gran medida de variables propias del docente como el género, la edad y el nivel académico, y del entorno tales como los recursos, la actitud de los directivos, los contenidos, entre otros (Riascos et al., 2012). Con relación a la edad, Vera et al. (2014) encontraron que a menor edad del docente se percibía un mayor dominio de las TIC y a mayor edad disminuyen sus conocimientos en relación a su uso. En el mismo sentido Nicholas y Guzmán (2009) reportan que aquellas personas nacidas después de 1980, pertenecientes a la denominada generación de los "Millennials" le dan una importancia superior a la autonomía laboral y evidenciaban mejores habilidades computacionales. En contraposición, Rosario y Vásquez (2012) desvinculan el uso e incorporación de las TIC a aspectos individuales, encontrándose que en relación a la formación en TIC para su uso y dominio en apoyo a las actividades académicas, los docentes en general muestran grandes deficiencias, independientemente de la edad, años dedicados a la docencia, nivel de escolaridad y su área de conocimiento.

Según su percepción frente al impacto de las TIC en la práctica docente y según su nivel de uso, los docentes se podrían clasificar en dos grandes grupos: aquellos docentes que se resisten a involucrase en las nuevas formas de enseñar, aduciendo que los métodos que han utilizado durante tanto tiempo han funcionado bien y no consideran necesario hacer cambios, y aquellos otros que, con mentalidad más abierta, están dispuestos a integrarse en este rol de docente innovador, en busca de mejorar las técnicas existentes de aprendizaje y explorar en el planteamiento de nuevos ideales pedagógicos (Riascos et al., 2012). Aunque surgen clasificaciones que matizan estas posturas opuestas, encontrándose profesores que podrían catalogarse en puntos intermedios entre los innovadores y los reticentes al cambio, como adoptantes tempranos, medios y tardíos (Santamaría et al., 2014).

Sarmiento y Guillén (2008) comentan que los modelos de formación docente, deben tomar en consideración aspectos tales como la adquisición de conocimiento disciplinar en su respectivo campo del saber, el desarrollo de habilidades relacionadas con el desempeño didáctico, además de un desarrollo integral del profesorado, su auto-concepto. Al respecto, Vera et al. (2014) reportan la existencia de una relación entre el nivel de adopción y el dominio de las habilidades asociadas a la aplicación educativa, evidenciándose que los docentes que se auto-perciben dentro de un nivel avanzado tienen mayor dominio en la aplicación de las tecnologías dentro del proceso de enseñanza-aprendizaje. Salanova (2003) expone que las personas persuadidas de que tienen las competencias para alcanzar con éxito determinadas acciones, tienen más probabilidad de realizar mayores esfuerzos y persistir en las adversidades que las personas que tienen dudas sobre las propias competencias. En el mismo sentido, distintas investigaciones han señalado la existencia de una tendencia general por parte del docente a considerar que posee una formación suficiente en aspectos relacionados con su manejo y utilización, pero insuficiente a la hora de llevar a cabo una integración profunda de las TIC en las actividades universitarias (Baelo, 2011). Una barrera crucial que hace que algunos académicos sean renuentes a comprometerse con las TIC en la educación es una carencia de confianza en sus propias habilidades tecnológicas y pedagógicas (Rienties et al., 2013).

Otro aspecto a considerar es el que atañe al estrés ocasionado por el uso de las tecnologías, aunque como lo menciona Salanova (2003) en sus investigaciones, la tecnología por sí misma es neutral, es decir, que no genera efectos ni positivos ni negativos, dado que la mera exposición a las TIC, no es la causa de efectos psicosociales negativos, sino que existen variables intervinientes que median y modulan esa relación, tales como la valoración de la experiencia pasada con el uso de las TIC, las actitudes y valores de la tecnología, y las creencias de autoeficacia en relación con el uso de la tecnología. 
Desde una aproximación comparativa, las diferencias evidenciadas entre los docentes universitarios en relación a la utilización y percepción de las TIC en la práctica docente según la naturaleza de la institución a la que se encuentran vinculados, pública o privada, se han reportado ciertas diferencias a favor de las instituciones privadas. Por ejemplo, en el contexto educativo venezolano, Rosario y Vásquez (2012) hallaron que los docentes de las instituciones privadas mostraron tener una mejor formación en TIC que los docentes de instituciones públicas, al igual que el disponer de un mejor equipamiento de las herramientas adecuadas y efectivas para hacer uso de las TIC en sus actividades académicas. En el mismo sentido, pero ya en el contexto colombiano, Riascos et al. (2012) reportan que la universidad privada presenta un interesante impacto de la utilización de las TIC, y le atribuye como explicación especialmente a la percepción positiva de la inclusión de las TIC en el proceso de enseñanza-aprendizaje y al alto nivel de utilización de las herramientas TIC.

La presente investigación se propuso identificar entre profesores universitarios el uso que realizan de las TIC en el desarrollo de su actividad profesional, así como recoger información sobre la percepción que tiene el profesorado con relación a la utilidad y relevancia de las TIC en los procesos de enseñanza y la valoración que hacen de los recursos tecnológicos disponibles en sus instituciones, desde una perspectiva comparativa entre profesores de universidades públicas y privadas.

\section{METODOLOGÍA}

La revisión de la literatura condujo a la construcción del instrumento que se planteó inicialmente bajo tres ejes temáticos, a saber: la percepción sobre las TIC por los profesores universitarios, el nivel de uso de las TIC en la práctica docente y la valoración de los docentes a los recursos tecnológicos disponibles en sus instituciones. Y como ítems complementarios en el instrumento, se recopilaron algunas características individuales del profesor. El cuestionario elaborado utilizó preguntas cerradas de opción de respuesta en escala ordinal y preguntas tipo Likert, en donde se realizaba una afirmación en la temática respectiva y se le solicitaba al docente seleccionar alguna de las opciones de respuesta en las modalidades de: totalmente en desacuerdo, en desacuerdo, neutro, de acuerdo y totalmente de acuerdo.

Este estudio hace uso de una invitación vía correo electrónico con un enlace directo a la encuesta (disponible en goo.gl/ay3158. Dicho instrumento fue diligenciado por profesores universitarios de instituciones públicas y privadas de las ciudades de Manizales, Pereira y Armenia, localizadas en la región del Eje Cafetero, Colombia. Los análisis fueron conducidos usando el software estadístico SPSS versión 18. El número de docentes encuestados fue de 147, de los cuales el $66 \%$ estaban vinculados a universidades públicas y el $34 \%$ a instituciones privadas. A continuación se presenta la tabla 1, con las frecuencias de distribución de la muestra para las distintas características individuales indagadas, acompañadas de los valores $\mathrm{P}$ de comparación, con el fin de constatar la no presencia de posibles sesgos en la conformación de los grupos estudiados.

Tabla 1: Conformación de la muestra: características descriptivas según naturaleza de la institución

\begin{tabular}{|l|l|l|c|}
\hline \multirow{2}{*}{ Variables } & \multicolumn{1}{|c|}{ Naturaleza de la universidad } & \multirow{2}{*}{ Valor $P$} \\
\cline { 2 - 5 } & \multicolumn{1}{|c}{ Pública } & \multicolumn{1}{|c}{ Privada } & 0.157 \\
\hline Género & Hombres (66\%)-Mujeres (34\%) & Hombres (54\%)-Mujeres (46\%) & 0.638 \\
\hline Edad & Antes 1981 (79.4\%)-Después 1980(20.6\%) & Antes 1981 (76\%)-Después 1980(24\%) & 0.085 \\
\hline Vinculación & De planta (61.9\%)-Otro (38.1\%) & De planta (76\%)-Otro (24\%) & 0.129 \\
\hline Escolaridad & $\begin{array}{l}\text { Prof/Esp (15.5\%)-Magíster (53.6\%)-Doctor } \\
(30.9 \%)\end{array}$ & $\begin{array}{l}\text { Prof/Esp (28\%)-Magíster (53.1\%)-Doctor } \\
(27.2 \%)\end{array}$ & 0.094 \\
\hline Experiencia & 15 años (-) (49.5\%)-15 años (+) (50.5\%) & 15 años (-) (64\%)-15 años (+) (36\%) & \multirow{2}{*}{ 15\%) } \\
\hline
\end{tabular}

El análisis contempló inicialmente la calibración del instrumento mediante la valoración de la validez de constructo del mismo y el análisis de consistencia interna. Para lo cual se procede al análisis factorial mediante el método de componentes principales y la obtención del indicador de fiabilidad haciendo uso del alfa de Cronbach. Para el análisis factorial se acude a las recomendaciones dadas en Hair et al. (1999) como se comenta a continuación. Se hace uso del análisis factorial tipo $\mathrm{R}$, el cual tiene como objetivo identificar la estructura de las relaciones entre las variables. Para verificar la adecuación del modelo se obtiene el KMO (0.753) y el contraste de esfericidad de Bartlett $(P=0.000)$, cuyos resultados indican que el análisis factorial es procedente para los datos recopilados. Para el cálculo del número de factores a ser extraídos se acude al criterio de la raíz latente, en el que se consideran los factores con autovalores superiores a 1, lo que conlleva a que sean retenidos 7 componentes con un porcentaje acumulado de varianza explicada del $64.6 \%$. Para mejorar la interpretación de los resultados se acude a una rotación factorial oblicua dado que los mismos no se consideran independientes. Y como criterio para la significación de las cargas factoriales se acude a la directriz basada en el tamaño muestral, que para el estudio realizado $(n=147)$ recomienda cargas factoriales mínimas de 0.5 . Los resultados del análisis se muestran en la tabla 2. 
Tabla 2: Componentes y estadísticos obtenidos del Análisis Factorial

\begin{tabular}{|c|c|}
\hline Componentes determinados a partir del Análisis Factorial & $\begin{array}{c}\text { Pesos } \\
\text { Factoriales }\end{array}$ \\
\hline \multicolumn{2}{|l|}{ Componente 1: Actividades on-line con los estudiantes (Autovalor=4.622; \%varianza=20.1) } \\
\hline \multicolumn{2}{|l|}{ Con qué frecuencia ha realizado las siguientes actividades on-line con sus estudiantes: } \\
\hline [Talleres/Trabajos ] & 0,817 \\
\hline [Evaluaciones ] & 0,786 \\
\hline [Tutorías] & 0,704 \\
\hline [Foros de discusión ] & 0,654 \\
\hline \multicolumn{2}{|c|}{ Componente 2: Satisfacción con los recursos tecnológicos de la institución (Autovalor=3.031; \%varianza=13.2) } \\
\hline \multicolumn{2}{|l|}{ Su opinión con respecto a las siguientes afirmaciones es: } \\
\hline [Los sistemas informáticos de la universidad son bastante seguros] & 0,852 \\
\hline [Me encuentro satisfecho con las conexiones a la red en los lugares de trabajo (aula, oficina,...] & 0,844 \\
\hline [La universidad dispone de una buena cobertura Wifi dentro de sus instalaciones] & 0,790 \\
\hline [La plataforma de conectividad de la universidad es adecuada] & 0,772 \\
\hline [La universidad provee una buena estructura tecnológica en el aula de clase] & 0,733 \\
\hline \multicolumn{2}{|l|}{ Componente 3: Herramientas de interacción emergentes (Autovalor=1.959; \%varianza=8.5) } \\
\hline \multicolumn{2}{|l|}{ Con qué frecuencia ha realizado las siguientes actividades on-line con sus estudiantes: } \\
\hline [Chats] & 0,814 \\
\hline [Comunicación por WhatsApp] & 0,737 \\
\hline [Videoconferencia] & 0,646 \\
\hline [Comunicación por Facebook] & 0,636 \\
\hline \multicolumn{2}{|l|}{ Componente 4: Habilidades TIC (Autovalor=1.487; \%varianza=6.5) } \\
\hline \multirow{3}{*}{$\begin{array}{l}\text { Su opinión con respecto a las siguientes afirmaciones es: } \\
\text { [Considero que tengo las habilidades requeridas para el uso de TIC en mis actividades laborales] } \\
\text { [Los inconvenientes en clase con las TIC los resuelvo con facilidad] }\end{array}$} & \\
\hline & 0,881 \\
\hline & 0,880 \\
\hline \multicolumn{2}{|l|}{ Componente 5: Herramientas clase (Autovalor=1.448; \%varianza=6.3) } \\
\hline \multicolumn{2}{|l|}{ En el desarrollo de sus clases utiliza: } \\
\hline [Video beam] & 0,747 \\
\hline [Archivos digitales] & 0,711 \\
\hline [Vídeo] & 0,685 \\
\hline [correo electrónico] & 0,561 \\
\hline \multicolumn{2}{|l|}{ Componente 6: Utilidad percibida de las TIC (Autovalor=1.260; \%varianza=5.5) } \\
\hline \multirow{4}{*}{$\begin{array}{l}\text { Su opinión con respecto a las siguientes afirmaciones es: } \\
\text { [Las herramientas TIC son primordiales en la enseñanza actual] } \\
\text { [El aprendizaje enriquecido por las TIC es más efectivo que los enfoques tradicionales] } \\
\text { [El uso de TIC en la docencia debe ir direccionado pedagógicamente] }\end{array}$} & \\
\hline & 0,835 \\
\hline & 0,724 \\
\hline & 0,669 \\
\hline \multicolumn{2}{|l|}{ Componente 7: Tecnoestrés (Autovalor=1.053; \%varianza=4.6) } \\
\hline Hay momentos en donde me siento estresado por el uso de la te & 0,917 \\
\hline
\end{tabular}

Como se puede observar los reactivos que indagan sobre la valoración que los docentes dan a los recursos tecnológicos disponibles en sus instituciones son acogidos en el componente 2; mientras que los que indagan sobre el uso de las TIC en la práctica docente, quedan agrupados en tres componentes, el componente 1, el 3 y el 5 , desagregados en actividades convencionales realizadas en línea, uso de herramientas de interacción emergentes y herramientas TIC empleadas en el aula de clase, respectivamente. Por su parte, las preguntas que indagaban sobre la percepción que los docentes tienen de 
las TIC quedan agrupadas en otros tres componentes, el 4 , el 6 y el 7 . Puntualmente, el componente 4 da cuenta de las preguntas de autovaloración sobre las capacidades de manejo de las TIC de los docentes, mientras que el componente 6 agrupa las variables sobre la utilidad percibida de las TIC por los profesores, y por último el componente 7 que registra una sola variable con una alta carga en dicho factor, la cual indaga sobre la sensación de estrés en los profesores ocasionada por el uso de la tecnología.

En cuanto a la medición de fiabilidad del instrumento, los resultados globales arrojan un valor del alfa de Cronbach de 0.774 , lo que indica que el instrumento empleado presenta un buen nivel de fiabilidad, con valores por dimensión por encima de 0.65 (Raykov, 2001), tal como queda registrado en la tabla 3.

Tabla 3: Valores del coeficiente alfa de Cronbach obtenido para los distintos componentes del instrumento

\begin{tabular}{|c|c|c|}
\hline Dimensiones & Número de elementos & Alfa de Cronbach \\
\hline 1 & 4 & 0.751 \\
\hline 2 & 5 & 0.860 \\
\hline 3 & 4 & 0.694 \\
\hline 4 & 2 & 0.761 \\
\hline 5 & 4 & 0.659 \\
\hline 6 & 3 & 0.693 \\
\hline 7 & 1 & N.A. \\
\hline Global & 23 & 0.774 \\
\hline
\end{tabular}

Posteriormente, y recurriendo a las puntuaciones factoriales para cada individuo generadas del análisis factorial, se procede a categorizar las dimensiones en valores altos y bajos según si dicha puntuación fuese positiva o negativa. Posterior a ello se realiza un Análisis de Correspondencias Múltiples (ACM en adelante) de tales dimensiones categorizadas junto a las variables descriptivas de los profesores. Finalmente se acude a la realización de pruebas de independencia con el propósito de evidenciar las diferencias en las respuestas dadas entre los profesores según la naturaleza de la institución a la cual se encuentra vinculada, es decir, si es pública o privada.

\section{RESULTADOS Y DISCUSIÓN}

Los resultados obtenidos arrojaron que con relación a los cuestionamientos sobre la percepción que los profesores tienen sobre las TIC, el $65.3 \%$ de los docentes encuestados consideran que preparar un curso en línea es más exigente que preparar un curso magistral, el $72.8 \%$ opinan que las herramientas TIC son primordiales en la enseñanza actual, el $54.4 \%$ declaran que el aprendizaje enriquecido por las TIC es más efectivo que los enfoques tradicionales, el $73.5 \%$ declaran estar de acuerdo con que los docentes presentan una información más efectiva si usan material audiovisual, mientras que el $22.4 \%$ de los profesores reconocen que han habido momentos en donde se han sentido estresados por el uso de las tecnologías. En cuanto a la formación en TIC, se encontró que $66.7 \%$ de los docentes considera que tienen las habilidades requeridas para el uso de las TIC en sus actividades laborales y el $49.7 \%$ de los docentes declara que resuelve con facilidad los inconvenientes que se presentan en clase con las TIC. En contraposición, se encuentra que el $24.5 \%$ de los encuestados considera que sus habilidades frente al manejo de las herramientas TIC son inferiores al de sus estudiantes.

Por otra parte, con relación a las preguntas sobre la frecuencia de las actividades en línea que el docente ha tenido con sus estudiantes, las que presentan mayores índices de uso son en su orden, el correo electrónico (98.6\%), la realización de talleres y trabajos (85\%), la realización de tutorías $(84.6 \%)$ y el uso del Whats App (69.7\%). Mientras que las de menor uso son el Facebook (37.4\%), las videoconferencias (49\%) y los foros de discusión (51.3\%). En este aspecto, los resultados coinciden con los obtenidos por Castillo et al. (2010) y Vera et al. (2014) en el contexto mexicano, quienes indican que un alto porcentaje de los docentes manifiestan usar con frecuencia las TIC en su labor docente y particularmente la mayoría de los docentes usan correo electrónico e internet con un promedio de cuatro veces por semana. Por su parte Morales et al. (2015) encontraron que la utilización de las TIC es cotidiana por parte de los docentes, aunque se evidencian vacíos en su integración para su práctica académica, a pesar de valorar la importancia que éstas ofrecen al proceso educativo y la creciente demanda que los alumnos hacen para su uso. Y en el mismo sentido, Riascos et al. (2012) comentan que algunos docentes tienen una percepción decidida a no utilizar las tecnologías argumentando diversos factores, tales como una deficiente preparación, o la falta de comprensión acerca de cómo la tecnología puede mejorar el currículum; además de ser docentes que sienten miedo de perder el control de su clase si utilizan alguna herramienta TIC dentro del proceso de enseñanza-aprendizaje.

Con el propósito de síntetizar la información, se acude al ACM con las dimensiones categorizadas en las modalidades altas y bajas, así como las variables descriptivas de los profesores utilizadas en este estudio. Tal como queda registrado en la figura 1, en donde se obtiene el Mapa Perceptual de dicho análisis, observándose como las variables naturaleza de la institución y género, acompañada de las dimensiones 
generadas en el análisis factorial, quedan cargando fuertemente al primer eje, discriminando a la izquierda los docentes de universidades públicas y a la derecha los de las privadas. Mientras que para el segundo eje quedan cargando las otras variables descriptivas del docente, como lo son, el tipo de vinculación con la institución, la edad, los años de experiencia y la escolaridad. Se observa entoces como a excepción de las variables naturaleza de la institución y género, las variables descriptivas muestran muy poca asociación con las variables de uso y percepción de las TIC. Por su parte, y realizando la lectura de los cuadrantes I y III del mapa, se observa como en el cuadrante I quedan las valoraciones altas a las dimensiones y por el contrario en el cuadrante III quedan sus contrapartes, las valoraciones bajas de las mismas. Es decir, los profesores de universidades privadas muestran una mayor asociación con un uso más alto de las TIC en la práctica docente, una valoración más alta a la infraestructura tecnológica en sus instituciones y una utilidad percibida más alta acerca de las TIC en la práctica docente. Pero de igual manera, manifiestan en mayor proporción haber sentido estrés por el manejo de las tecnologías. Y para el cuadrante III, en donde su localizan los profesores de universidades públicas, se ubican las modalidades de respuesta opuestas.

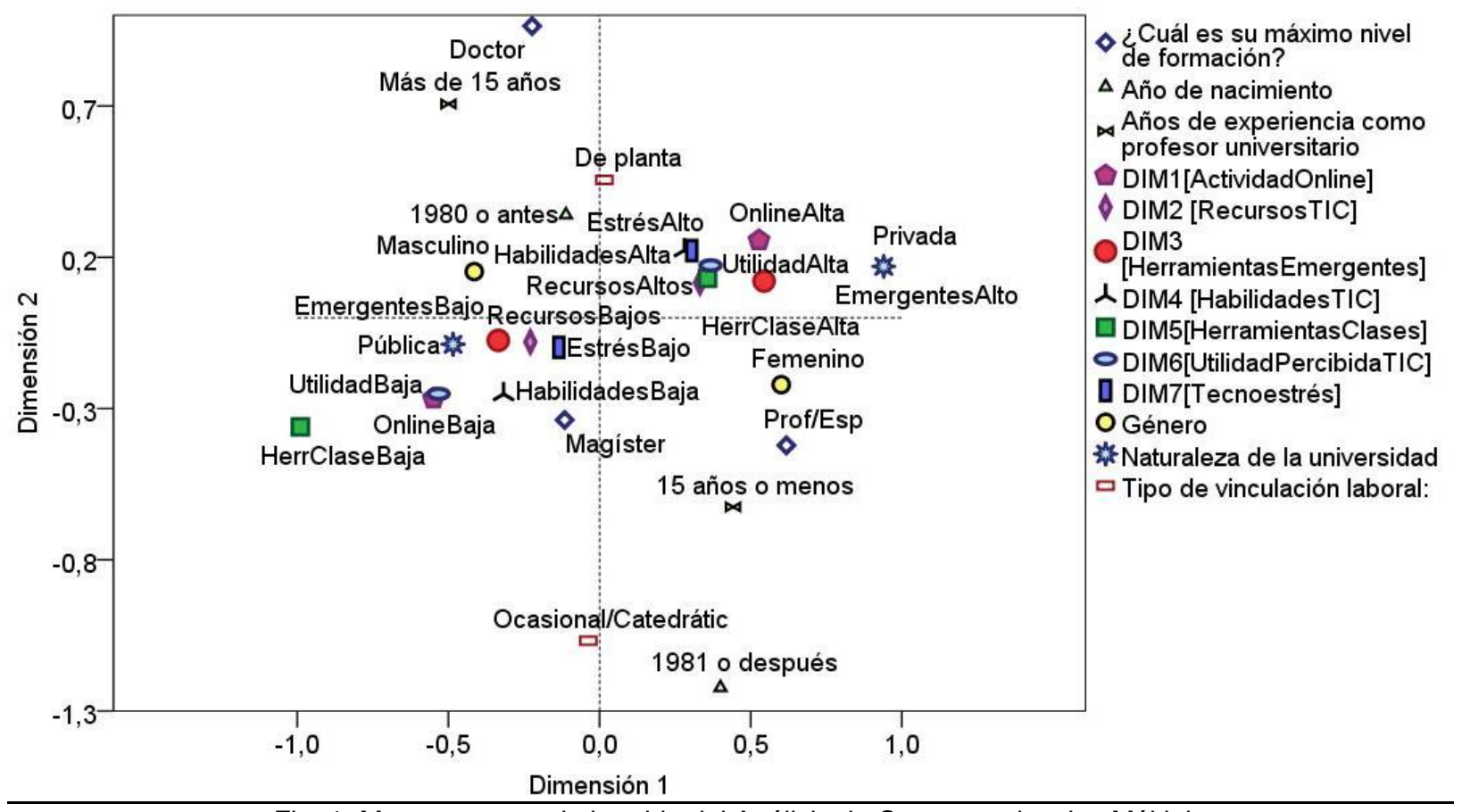

Fig. 1: Mapa perceptual obtenido del Análisis de Correspondencias Múltiples

Ahondando en los resultados obtenidos, se obtiene mediante la aplicación de las pruebas de independencia chi-cuadrado que a un nivel de confianza del $95 \%$ no se detectan diferencias significativas entre profesores de universidades públicas y privadas con respecto al uso de los siguientes medios telemáticos usados en clase: correo electrónico $(P=0.101)$, comunicación por Facebook $(P=0.693)$, tutorías $(P=0.276)$ y chats $(P=0.128)$. En contraste, si se detectan diferencias significativas a favor de los profesores de universidades privadas en actividades en línea realizadas con los estudiantes por WhatsApp $(P=0.000)$, la realización de evaluaciones en línea $(P=0.000)$, la realización de talleres y trabajos $(P=0.011)$, la realización de foros de discusión $(P=0.001)$ y la realización de actividades mediante videoconferencias $(P=0.011)$.

Es importante precisar que también se realizaron las pruebas de independencia sobre el uso de los medios telemáticos en la actividad docente para los profesores nacidos antes de 1981 y los nacidos después de 1980, y contrario a lo hallado por Nicholas y Guzman (2009) y Vera et al. (2014), no se encontraron diferencias significativas para ninguno de los medios electrónicos entre los dos grupos referenciados. Pero consistentes con los hallazgos de Castillo et al. (2010) quienes declaran que aunque los profesores de licenciatura son más jóvenes, no necesariamente utilizan con mayor frecuencia las TIC. De similar forma se procedió para el análisis comparativo según el género del profesor, encontrándose diferencias significativas en algunos ítems a favor de las mujeres. Puntualmente, se encontró que las profesoras hacían un mayor uso del WhatsApp en actividades académicas que los hombres ( $P=0.043$ ), con un $45 \%$ de las mujeres contra un $28.7 \%$ de los hombres; en el mismo sentido las docentes hacen más uso de las tutorías en línea $(\mathrm{P}=0.011)$ con un $68.3 \%$ contra un $47.1 \%$ de los hombres; y en la realización de videoconferencias $(\mathrm{P}=0.016)$, con un $18.3 \%$ contra un $5.7 \%$ de los hombres.

Ahora, con relación a las percepciones manifestadas por los docentes universitarios respecto al rol de las TIC en la actividad docente actual, se observa que, tal como queda registrado en la tabla 4 , no se detectan 
diferencias significativas entre las consideraciones de los profesores de universidades públicas y privadas. A excepción de lo expresado con respecto a considerar que el aprendizaje enriquecido por las TIC es más efectivo que los enfoques tradicionales, en donde casi tres cuartas partes de los profesores de instituciones privadas manifiestan estar de acuerdo con dicha afirmación, mientras que para los docentes de instituciones públicas menos de la mitad está alineado con tal afirmación; al igual que en las repuestas sobre el haber sentido estrés relacionado por el uso de la tecnología, en donde la proporción de docentes de universidades privadas, prácticamente duplican a la proporción de docentes de universidades públicas en este ítem.

Tabla 4: Resumen de resultados de las pruebas de independencia con respecto a percepciones sobre uso de las TIC según la naturaleza de la universidad

\begin{tabular}{|c|c|c|c|}
\hline \multirow[t]{2}{*}{ Afirmaciones } & \multicolumn{2}{|c|}{$\begin{array}{l}\text { Naturaleza de } \\
\text { la universidad }\end{array}$} & \multirow[b]{2}{*}{ Valor $P$} \\
\hline & Pública & Privada & \\
\hline Considero que las herramientas TIC son primordiales en la enseñanza actual & $69.1 \%$ & $80.0 \%$ & 0.158 \\
\hline $\begin{array}{l}\text { El aprendizaje enriquecido por las TIC es más efectivo que los enfoques } \\
\text { tradicionales }\end{array}$ & $45.4 \%$ & $72.0 \%$ & 0.002 \\
\hline Pienso que el uso de TIC en la docencia debe ir direccionado pedagógicamente & $90.7 \%$ & $90.0 \%$ & 0.888 \\
\hline Los docentes presentan una información más efectiva si usan material audiovisual & $69.1 \%$ & $82.0 \%$ & 0.093 \\
\hline Hay momentos en donde me siento estresado por el uso de la tecnología & $17.5 \%$ & $32.0 \%$ & 0.046 \\
\hline
\end{tabular}

Igualmente, se realiza un análisis de la opinión registrada por los docentes con relación a la infraestructura tecnológica que las instituciones les proporcionan en sus instalaciones según la naturaleza de la universidad a la que se encuentra vinculado, ya sea pública o privada. Como queda registrado en la tabla 5 , se observan diferencias estadísticamente significativas $(P<0.05)$ a favor de los docentes de instituciones privadas con relación a considerar que la universidad provee una buena infraestructura tecnológica en el aula de clase; encontrarse satisfecho con las conexiones a la red en los lugares de trabajo (aula, oficina, laboratorio); disponer de una buena cobertura Wifi dentro de sus instalaciones y considerar que los sistemas informáticos de la universidad son bastante seguros.

Tabla 5: Resumen de resultados de las pruebas de independencia con respecto a la infraestructura tecnológica disponible según la naturaleza de la universidad

\begin{tabular}{|l|c|c|c|}
\hline \multicolumn{1}{|c|}{ Afirmaciones } & \multicolumn{2}{c|}{$\begin{array}{c}\text { Naturaleza de } \\
\text { la universidad }\end{array}$} & \multirow{2}{*}{ Valor P } \\
\cline { 2 - 3 } & Pública & Privada & Valor \\
\hline La universidad provee una buena estructura tecnológica en el aula de clase & $32,0 \%$ & $64,0 \%$ & 0.000 \\
\hline $\begin{array}{l}\text { Me encuentro satisfecho con las conexiones a la red en los lugares de trabajo } \\
\text { (aula, oficina, laboratorio, ...) }\end{array}$ & $19.6 \%$ & $50.0 \%$ & 0.000 \\
\hline La universidad dispone de una buena cobertura Wifi dentro de sus instalaciones & $19.6 \%$ & $48.0 \%$ & 0.000 \\
\hline Considero que los sistemas informáticos de la universidad son bastante seguros & $25.8 \%$ & $56.0 \%$ & 0.000 \\
\hline
\end{tabular}

Es importante reseñar que es lógico suponer, tal como lo sugieren Ávila y Riascos (2011) que la valoración registrada por los docentes acerca de la infraestructura tecnológica institucional esté matizada por las políticas institucionales relativas a las estrategias para la aplicación de las TIC y su uso efectivo en el ámbito de la institución, aspecto que no es considerado en el presente estudio.

\section{CONCLUSIONES}

A la luz de los resultados obtenidos se observa como las herramientas tecnológicas se han ido lentamente incorporándo a la actividad docente, y cómo la percepción que los docentes tienen del rol de las TIC y de su importancia en las actividades académicas inciden en la apropiación de dichas herramientas. Es así como se observan diferencias favorables a los profesores de instituciones privadas con relación a la percepción, apropiación y uso que éstos tienen de las herramientas TIC en la práctica docente. Puntualmente, los docentes de universidades privadas consideran en una mayor proporción, que el aprendizaje enriquecido por las TIC es más efectivo que los enfoques tradicionales de enseñanza. También se evidencia un mayor uso de los medios telemáticos para entablar comunicación con sus estudiantes en actividades en línea, tales como la comunicación por WhatsApp, la realización de evaluaciones, talleres y trabajos en línea, la realización de foros de discusión y videoconferencias.

En igual sentido, se evidencian diferencias significativas favorables a las universidades privadas con respecto a la valoración que sus docentes hacen de la infraestructura tecnológica disponible para la realización de su actividad docente. Pero es de reseñar, las diferencias obtenidas con relación a la sensación de estrés percibida por el uso de la tecnología en la labor docente, registrándose que aproximadamente uno de cada tres profesores de universidades privadas manifiestan haberla sentido, 
mientras que en las públicas esta proporción se reduce casi a la mitad. A manera de conjetura, y necesitándose evidencia empírica al respecto, se podría percibir que un mayor uso de las TIC en las universidades privadas se deba más a directrices de los directivos de estas instituciones que a una decisión espontánea de los docentes. Por otro lado, y al menos para la población estudiada, no se detectó una incidencia de la generación al cual pertenece el docente, al menos en los denominados Millenials no se detectaron comportamientos diferentes en relación a las variables estudiadas con respecto a los otros profesores, nacidos antes de 1981. Mientras que si se registraron diferencias significativas según el género del docente, obteniéndose resultados muy favorables en el grupo de las mujeres, puntualmente en relación a un mayor uso de las herramientas tecnológicas en actividades académicas.

Para finalizar, los resultados muestran que a nivel de las instituciones públicas, hay cierto grado de disconformidad entre los profesores con relación a la infraestructura tecnológica de sus instituciones, lo que contrasta con las inversiones realizadas en los últimos años por los gobiernos de turno para el fortalecimiento de la infraestructura tecnológica, por lo que sería necesario indagar las causas de tal percepción entre los docentes. Análogamente, para las instituciones privadas, indagar las causales de la presión percibida por una parte significativa de sus docentes, en procura de llevar a cabo procesos formativos consensuados y avalados por los distintos estamentos universitarios.

\section{AGRADECIMIENTOS}

Los autores agradecen a la Universidad Nacional de Colombia sede Manizales a través de la dirección de investigaciones (DIMA) por el apoyo institucional y financiero al desarrollo de esta investigación (proyecto: "Limitaciones y barreras de los empresarios manizaleños frente a la adopción del teletrabajo en sus empresas" código Hermes 28596).

\section{REFERENCIAS}

Ausín, V., Abella, V., Delgado, V. y Hortigüela, D. Aprendizaje Basado en Proyectos a través de las TIC. Una Experiencia de Innovación Docente desde las Aulas Universitarias. doi: 10.4067/S071850062016000300005, Form. Univ., 9(3), 31-38 (2016)

Ávila-Fajardo, G., Riascos-Erazo, S. Propuesta para la medición del impacto de las TIC en la enseñanza universitaria. ISSN: 0123-1294, Revista Educación y Educadores, 14(1), 169-188 (2011)

Baelo, R. Satisfacción del profesorado universitario con la integración de las tecnologías de la información y la comunicación (TIC). ISSN: 1695-324X, Revista Etic@net, (11), 253-276 (2011)

Baelo, R. y Cantón, I. Las tecnologías de la información y la comunicación en la educación superior. Estudio descriptivo y de revisión. ISSN: 1681-5653, Revista Iberoamericana de Educación, 50(7), 1-12 (2009)

Cabero, J. Las TICs y las Universidades: retos, posibilidades y preocupaciones. ISSN 0185-2760, Revista de la Educación Superior, 34(3), 77-100 (2005)

Cabero, J., Llorente, M.C., Puentes, A., Marín, V., Cruz, I. La competencia digital del profesorado: un estudio en la Pontifica Universidad Católica Madre y Maestra. ISBN: 978-84-936798-7-3, Edita: Grupo de investigación Didáctica de la Universidad de Sevilla, Sevilla, España (2011)

Cantón, I., Baelo, R. El profesorado universitario y las tecnologías de la información y de la comunicación (TIC): disponibilidad y formación. ISSN: 1989-466X, Educatio Siglo XXI, 29(1), 263-302 (2011)

Castillo, M., Larios, V. y Ponce, O. Percepción de los docentes de la utilización de las Tecnologías de la Información ay la Comunicación. ISSN: 1681-5653, Revista Iberoamericana de Educación, 53(6), 1-10 (2010)

Correa, J. y Paredes, J. Cambio tecnológico, usos de plataformas de e-learning y transformación de la enseñanza en las universidades españolas: la perspectiva de los profesores. ISSN: 2254-4372, Revista de Psicodidáctica, 14(2), 261-278 (2009)

Departamento Nacional de Planeación (DNP) de Colombia. Reporte Global de Tecnologías de la Información 2015-Síntesis de resultados para Colombia, (en línea: https://goo.gl/s2vFbT, acceso 2 de noviembre de 2016), DPN Colombia (2015)

Duart, J., Reparaz, Ch. Enseñar y aprender con la TIC. ISSN: 1578-7001, Estudios sobre educación, 20(1), 9-19 (2016) 
Hair, J., Anderson, R., Tatham, R., Black, W. Análisis Multivariante, $5^{a}$ edición, 79-121, Prantice Hall lberia, Madrid, España (1999)

Jones, A. Teacher Perceptions and Use of ICT in Unfamiliar Classroom Situations. ISBN: 978-99957-0-2441 ICICTE 2012 Proceeding, 314-322, Rhodes, Grecia, Julio 5-7 (2012)

Kirkwood, A., Price, L. Adaptation for a Changing Environment: Developing learning and teaching with information and communication technologies. ISSN: 1492-3831, International Review of Research in Open and Distance Learning, 7(2), 1-14 (2006)

Lagunes-Domínguez, A., Torres-Gastelú, C., Flores-García, M. y Rodríguez-Figueroa, A. Comparativo del uso de Tecnologías de la Información y Comunicación (TIC) por Profesores de Dos Universidades Públicas de México. doi: 10.4067/S0718-50062012000500002, Form. Univ., 8(2), 11-18 (2015)

Ministerio de Educación Nacional de Colombia (MEN). La innovación educativa en Colombia. Buenas prácticas para la innovación y las TIC en Educación, (en línea: https://goo.gl/UvQ1Mm, acceso: 2 de noviembre de 2016), MEN Colombia (2016)

Muñoz-Cano, Juan M., Córdova, Juan A. y Priego, Heberto. Dificultades y facilidades para el desarrollo de un proceso de innovación educativa con base en las Tecnologías de la Información y Comunicación (TIC). doi: 10.4067/S0718-50062012000500002, Form. Univ., 5(1), 3-12 (2012)

Morales, M., Trujillo, J., Raso, F. Percepciones acerca de la integración de las TIC en el proceso de enseñanza-aprendizaje de la Universidad. ISSN: 1133-8482, Revista de Medios y Educación, 46(1), 103-117 (2015)

Nicholas, A., Guzman, I. Is Teleworking for Millennials? Proceedings of the 2009 ACM SIGMIS CPR Conference on Computer Personnel Research, Limerick, Ireland, May 28-30 (2009)

Raykov, T. On the Use and Utility of the Reliability Coefficient in Social and Behavioral Reseach, doi: 10.1023/A:1010381529095, Quality and Quantity, 35(1), 253-263 (2001)

Riascos, S., Ávila, G., Quintero, D. Las TIC en el aula: percepciones de los profesores universitarios. ISSN: 0123-1294, Revista Educación y Educadores, 12(3), 133-157 (2012)

Rienties, B., Brouwer, N., Lygo, S. The effects of online professional development on higher education teachers' beliefs and intentions towards learning facilitation and technology. ISSN: 0742-051X, Teaching and Teacher Education, 29(1), 122-131 (2013)

Rosario, H., Vásquez, L. Formación del docente universitario en el uso de TIC. Caso universidades públicas y privadas (U. de Carabobo y U. Metropolitana). ISSN: 1133-8482, Revista de Medios y Educación, (41), 163-171 (2012)

Salanova, M. Trabajando con tecnologías y afrontando el tecnoestrés: el rol de las creencias de eficacia. ISSN: 1576-5962, Revista de Psicología del Trabajo y de las Organizaciones, 19(3), 225-246 (2003)

Santamaría, M., San Martín, S., López, B. Perfiles de alumnos según el uso deseado de las TIC por el profesor universitario. ISSN: 1133-8482, Revista de Medios y Educación, (45), 37-50 (2014)

Sarmiento, M., Guillén, J. Formación en TIC: necesidades del profesor universitario. ISSN: 1315-883X, Laurus Revista de Educación, 14(28), 11-34 (2008)

Vera, J., Torres, L. y Martínez, E. Evaluación de competencias básicas en TIC en docentes de educación superior en México. ISSN: 1133-8482, Revista de Medios y Educación, (44), 143-155 (2014)

Yong Varela, L.A., Rivas Tovar, L. A. y Chaparro, J. Modelo de aceptación tecnológica (TAM): un estudio de la influencia de la cultura nacional y del perfil del usuario en el uso de las TIC. ISSN: 0121-5051, Innovar, 20(36), 187-204 (2010) 